\title{
Inoculando los valores de la Economía Social en las empresas convencionales
}

Inculcating the values of the Social Economy in conventional enterprises Juan Manuel Sinde Oyarzabal*

Presidente. Arizmendiarrieta Kristau Fundazioa

* Correspondencia a/Corresponding author: Juan Manuel Sinde Oyarzabal. Arizmendiarreta Kristau Fundazioa - juanmasinde@gmail.com

Cómo citar/How to cite: Sinde Oyarzabal, Juan Manuel (2021). «Inoculando los valores de la Economía Social en las empresas convencionales", GIZAEKOA - Revista Vasca de Economía Social, 18, 303-313. (https://doi.org/10.1387/gizaekoa.23098).

Recibido: 20/05/2021; aceptado: 27/05/2021.

ISSN 1698-7446 - eISSN 2444-3107 / (c) 2021 UPV/EHU

(c) (†) Esta obra está bajo una licencia

Creative Commons Atribución 4.0 Internacional 


\section{Introducción}

Como es sabido la Economía Social se define en el estado español por criterios jurídicos. Forman parte de la misma la siguiente tipología de organizaciones: cooperativas, sociedades laborales, mutualidades, empresas de inserción, cofradías de pescadores y centros especiales de empleo.

Esta clasificación no coincide con la existente en otros países europeos, que, en ocasiones, no tiene una tipología jurídica sino que está caracterizada por una serie de valores diferenciales de las organizaciones que en ella se agrupan.

Con ese marco, vamos a tratar de buscar la aplicación de esos valores en las empresas convencionales, tratado de trascender los límites jurídicos para «inocularlos» en empresas que no forman parte del perímetro de la Economía Social, como forma de hacerlas no sólo más humanas sino también más competitivas en el escenario económico actual.

El trabajo se divide en los siguientes apartados:

1. Introducción.

2. Los valores de la Economía Social.

3. Razones desde la competitividad para unas empresas más humanas.

4. Características del Modelo inclusivo participativo de empresa (MIPE).

5. Algunas políticas públicas para su promoción y desarrollo.

6. Conclusiones.

\section{Los valores de la Economía Social}

Social Economy Europe, institución que agrupa a las distintas realidades de la Economía Social en los países de la UE, ha definido los valores que considera característicos de las empresas que se reclaman de esa categoría. Son los siguientes:

- Primacía de las personas y de los objetivos sociales frente al capital.

- Gobernanza democrática y participativa.

— Libre adhesión.

-Equilibrio entre los intereses de sus miembros y el interés general de la sociedad.

- Promoción y aplicación de los principios de solidaridad y responsabilidad.

- Gestión autónoma e independiente de los poderes públicos.

- Reinversión de la mayor parte de los beneficios para conseguir los objetivos de un desarrollo sostenible y responder al interés de sus miembros y de la sociedad. 
Además de esos valores, la Economía Social se caracteriza por un fuerte enraizamiento en las comunidades en las que las empresas y organizaciones operan.

Todos ellos serían la base sobre la que articular unas posibles empresas más humanas, cuyas motivaciones desde la competitividad y sus características concretas se desarrollan en los próximos apartados.

\section{Razones desde la competitividad para unas empresas más humanas}

Siguiendo las reflexiones de varios grupos de personas representativas de las diversas sensibilidades económicas, políticas y sociales de la CAPV, Navarra y el conjunto del Estado, destacaríamos, en primer lugar, las características del escenario en el que las empresas desarrollan su actividad.

Así, merece subrayar que, en las últimas décadas, dos han sido los factores más importantes que han modificado el escenario competitivo para las empresas vascas, españolas y europeas en general: el acelerado avance de los descubrimientos científicos y sus aplicaciones técnicas y la globalización de los procesos económicos de producción, distribución, financiación y consumo.

Con ese marco, las razones para unas empresas más humanas serían las siguientes:

1. Como consecuencia del vertiginoso desarrollo técnico citado ha cambiado radicalmente el papel de las personas en las empresas, siendo de vital importancia la adecuada gestión del conocimiento y de la innovación, que reside precisamente en dichas personas.

2. Éstas tienen un papel crítico en la competitividad y el éxito de todo tipo de empresas al aportar su inteligencia, inquietudes, creatividad, empatía y capacidad de trabajar en equipo. Se trata de un factor de primerísima importancia desde el punto de vista empresarial que habría que cuidar, motivar, mejorar...

3. Esta situación genera, por consiguiente, la necesidad de situar a los equipos humanos en el centro de la gestión de las empresas, no sólo, por tanto, por razones humanistas sino también por razones de eficiencia y competitividad.

4. Por otro lado, el proceso de globalización exige que las empresas y los países que no pueden competir vía costes tienen que buscar otros elementos de competencia, basados en la mejora constante 
de la calidad y en nuevas propuestas de valor para sus clientes que, a su vez, dependen de las personas, lo que refuerza la importancia de las mismas.

5. Pero para asegurar su papel principal se precisa, en ese contexto, un nuevo modelo de empresa que facilite y potencie el conocimiento y la implicación de todos los agentes en un proyecto empresarial compartido.

6. El cambio, por otro lado, debe construirse desde la confianza, cimentada en una información transparente, veraz y sistemática y en un modelo de gestión que fomente la participación en el «día a día». Esta confianza debe compartirse entre la comunidad de la empresa, los colectivos con los que se relaciona y las Administraciones Públicas.

7. Es necesario, por tanto, introducir cambios culturales y organizativos en las empresas, lo que implica adaptar de forma significativa el modelo de relación tradicional entre empresarios y trabajadores, con el fin de formular un proyecto común que influya positivamente tanto en la competitividad y creación de valor para la empresa como en alcanzar unas condiciones salariales y de trabajo dignas para todas las personas implicadas.

8. Por lo que resulta esencial preocuparse de que éstas tengan un salario digno, una formación de futuro, su adhesión al proyecto empresarial, la satisfacción en el trabajo, la cohesión social que facilita el trabajo en equipo, un trato justo desde el punto de vista de género, la preocupación por la conciliación de la vida profesional y familiar...

9. El cambio que se propone implica entender los objetivos de las empresas de forma amplia y no sólo como la búsqueda del máximo beneficio a corto plazo. Se debería avanzar en fórmulas de éxito internacional en las que la empresa se concibe como un organismo que satisface de forma equilibrada una constelación de intereses, a la vez que tiene en cuenta también cuestiones ecológicas, de Derechos Humanos...

10. La responsabilidad del cambio es de todos, pero en especial y en mayor medida de los que detentan el poder en la empresa, destacándose la importancia de la ejemplaridad de los empresarios y directivos y el avance en el cambio cultural hacia el trabajo en equipo, la honestidad en las relaciones y la apertura a los cambios necesarios para la sostenibilidad de la empresa.

11. En un contexto en el que la experiencia indica que para promover la participación es necesario generar condiciones de entorno 
favorables por parte de las instituciones públicas y los agentes económicos y sociales, en el sentido de favorecer un modelo socio-productivo que prime la sostenibilidad social, económica y medioambiental y el interés colectivo respecto al interés individual.

12. Subrayando, con todo, que el cambio hacia este nuevo modelo no sólo es cuestión de normativa legal, ni es automático: es necesario gestionar y trabajar internamente la cultura empresarial, lo que exige en primer lugar el compromiso de los máximos dirigentes de la empresa para que la nueva cultura se diseñe y organice de manera que logre la participación y el compromiso de todos los trabajadores y de sus representantes.

13. Implica, por tanto, la iniciativa de directivos y empresarios, responsables de iniciar el cambio, aunque para la implementación efectiva de ese modelo de participación necesitará concitar las adhesiones de las personas que forman parte de la empresa y el interés de sus representantes, aprovechando las competencias de información y consulta que se atribuyen a los Comités de Empresa en el artículo 64 del Estatuto de los Trabajadores.

\section{Características del Modelo inclusivo participativo de empresa (MIPE)}

La presente propuesta del Modelo inclusivo participativo de empresa 2020 parte del texto de la propuesta de la Proposición No de Ley presentada el 15-5-18 por todos los grupos políticos representados en el Parlamento Vasco y posteriormente aprobada por unanimidad en setiembre del mismo año.

Las modificaciones y matizaciones que recogemos en esta Aplicación 2020 para la CAV tienen las siguientes fuentes:

1. Algunas aportaciones del Parlamento navarro sobre los ítems que figuran en el texto inicial.

2. Algunas aportaciones recogidas en las numerosas presentaciones realizadas a grupos muy diversos con posterioridad a la aprobación parlamentaria (en especial, a diversas asociaciones empresariales y sindicatos relevantes, tanto vascos como europeos.

3. Las reflexiones realizadas en el Seminario organizado por la Fundación Arizmendiarrieta y Bilbao Metrópoli 30 con un grupo de empresarios, directivos y consultores de Bizkaia. 
Teniendo en cuenta estos nuevos inputs, el Modelo inclusivo participativo tendría, en esta fase, las siguientes características:

1. Una cultura de cooperación, corresponsabilidad y preocupación por las personas (frente al paradigma de confrontación como actitud básica), que se materializaría en:

a) Crear un clima de confianza mediante una política de transparencia informativa, con información regular y regulada a accionistas y trabajadores sobre las variables y políticas más importantes de la empresa, salvando aquellas que pudieran ser estrictamente confidenciales.

b) Programar y desarrollar planes de formación sistemáticos con objetivos de dedicación por trabajador e incluyendo en los mismos tanto formación técnica como formación de gestión que permita una participación efectiva en un proyecto compartido y la formación continua. Como referencia, existiría un objetivo de una semana de formación al año por persona, llegando anualmente a un mínimo del $80 \%$ de los trabajadores de la plantilla.

c) Impulsar políticas retributivas de trabajadores, técnicos y directivos de forma que, salvando la necesaria fidelización de los profesionales con habilidades críticas para el proyecto empresarial, no generen una gran desigualdad y favorezcan la cohesión social, siendo recomendable, por otro lado, que la remuneración incorpore alguna participación en resultados susceptible de alcanzar a la totalidad de la plantilla.

d) Establecer sistemas periódicos de evaluación y mejora continua de la satisfacción y necesidades de las personas que en ella trabajan, implicando para ello tanto a la vía ejecutiva como a representantes de los trabajadores. Una referencia podría ser realizar un estudio sistemático con una frecuencia bienal.

e) Priorizar, sin exclusividad, la promoción interna para asignar funciones de mayor responsabilidad y utilizar criterios objetivos de valoración del mérito y posibilidades de aportación a la hora de seleccionar entre los diversos candidatos para ocupar puestos de responsabilidad.

f) Eliminación de la brecha salarial de género.

g) Buscar fórmulas que favorezcan la corresponsabilidad de género y la conciliación de la vida profesional y familiar de todos sin perjuicio del desarrollo empresarial. 
2. La participación de los trabajadores en la gestión y/o en los resultados y/o en la propiedad (frente a la separación radical de capital y trabajo), que implicaría:

a) Implantar sistemas de gestión participativos, con procedimientos y herramientas adecuados, lo que incluiría consultar con los representantes de los trabajadores las decisiones más relevantes de la empresa que no exijan un tratamiento confidencial y estableciendo protocolos para ello si fuera necesario.

b) Estudiar el compromiso de repartir un \% de sus resultados entre todos los componentes de su plantilla. La consecución de este objetivo podrá alcanzarse en un plazo de 5 años.

3. La prioridad de la sostenibilidad del proyecto colectivo sobre los intereses de cualquiera de los grupos de interés (frente a la prioridad de los intereses parciales de unos y otros):

a) Priorizar estrategias de crecimiento sostenible sobre estrategias de rentabilidad a corto plazo, buscando crecimientos del volumen de negocio que, en función de la situación del mercado, favorezcan la creación y mantenimiento de puestos de trabajo de forma sostenible.

b) Plantear objetivos anuales que tangibilicen las ventajas del nuevo modelo, mejorando a la vez la rentabilidad sobre fondos propios para los accionistas y la retribución global para los trabajadores

c) Destinar al menos un 50\% de los beneficios anuales a incrementar los Fondos Propios, de forma que facilite la inversión en equipos, actividades de $\mathrm{I}+\mathrm{D}+\mathrm{i} \mathrm{y}$, consiguientemente, su desarrollo y sostenibilidad. La consecución de este objetivo de $50 \%$ sería de media y debería alcanzarse en un plazo de 5 años.

d) Destinar a actividades de $\mathrm{I}+\mathrm{D}$ y formación un porcentaje sobre ventas superior a la media del sector.

4. Tener en cuenta las necesidades de la comunidad en la que se asienta (frente a la separación radical de actividad empresarial y responsabilidad social):

a) Respetar y cumplir rigurosamente la legislación, especialmente la laboral y medioambiental, de los países en los que desarrolle su actividad, así como las recomendaciones de la OIT.

Mantener una política de honestidad fiscal, evitando el fraude y la utilización de paraísos fiscales, de forma que no se vean mer- 
mados los recursos públicos necesarios para afrontar los retos económicos y sociales de la Comunidad.

b) Dedicar un porcentaje del 1\%-3\% de los beneficios a actividades de Responsabilidad Social valorando la implicación de los trabajadores en los distintos proyectos y contando para todo ello con el Comité de Empresa. La consecución de este objetivo debería alcanzarse en un plazo de 5 años.

c) Participar en la reflexión, evaluación y, si cabe, implementación de las políticas sociales dirigidas a la integración laboral de los trabajadores menos cualificados que puedan quedar «excluidos» de los procesos productivos por efecto de la incorporación de las nuevas tecnologías o de su situación personal.

d) Colaborar con los órganos públicos correspondientes para la adecuación permanente de la FP, la formación continua y la formación universitaria a las necesidades de las empresas, estableciendo los planes de formación dual, experiencia en prácticas, programas de servicio y aprendizaje adecuados. En definitiva, una comunicación permanente entre el mundo empresarial y el formativo para construir un proyecto común.

\section{Algunas políticas públicas para su promoción y desarrollo}

El cambio debe ser favorecido por los poderes públicos y alentado por la sociedad en general. Se considera, de forma especial, que la Administración Pública debe involucrarse para estimular la reinversión de una parte significativa de los resultados obtenidos, así como para favorecer la innovación, el desarrollo y la sostenibilidad de las empresas a largo plazo.

En el presente documento recogemos dos bloques de medidas:

1. Las aprobadas en los Parlamentos vasco y navarro, que hemos adaptado teniendo en cuenta reflexiones y experiencias posteriores, así como la posibilidad de plantearlas en los Organos de la Admon. Central del Estado, con competencias que superan las que tienen los Órganos autonómicos en materia de regulación de la actividad económico-empresarial.

2. Otras recibidas de distintas fuentes y que entendemos no suscitan rechazo de los agentes sociales.

Las clasificamos en los grupos siguientes:

- Grupo I: Medidas para la generación de conocimiento, concreción y divulgación del el nuevo Modelo. 
—Grupo II: Posibles cambios normativos y legales. Las medidas concretas serían las siguientes:

\section{Grupo I. Medidas para la generación de conocimiento, concreción y divulgación del el nuevo Modelo}

1. Colaborar en la elaboración de un Modelo de Evaluación de la implantación del Modelo inclusivo-participativo, con un "check list» que ayude a las empresas a identificar los avances registrados.

2. Desarrollar actividades de Formación sobre aspectos de gestión empresarial, estrategia, ... dirigidas a personas componentes de Comités de Empresa, delegados sindicales, liberados de Sindicatos..., tanto de forma especial como de forma conjunta con directivos de las empresas implicadas.

3. Profundizar, apoyándose preferentemente en las Asociaciones empresariales, en el apoyo a la difusión e implementación de sistemas participativos de gestión característicos del modelo inclusivo-participativo, ampliándolo a la definición e implantación de las Mejores Prácticas en el establecimiento de políticas de comunicación interna en las empresas, definición de objetivos, selección de indicadores, ..., que se considera clave para el cambio propuesto.

4. Colaborar en campańas de sensibilización dirigida a empresarios y a trabajadores para fomentar el modelo inclusivo-participativo propuesto.

5. Estimular igualmente la implantación de sistemas de participación de los trabajadores en los resultados de la empresa, colaborando con las citadas Asociaciones empresariales en la identificación de las fórmulas de mayor éxito tanto en Espańa como a nivel europeo.

6. Prestigiar a las empresas que destaquen en la implantación del modelo mediante la organización de algún premio o reconocimiento al respecto.

7. Apoyar la creación de un servicio de asesoramiento en las asociaciones de empresarios, de las empresas familiares...

8. Identificar las Mejores Prácticas a nivel internacional en lo que se refiere a políticas fiscales que ayuden a promover la participación en la propiedad.

9. Estudiar la creación de un Instituto, con la colaboración de las Asociaciones empresariales y la Admon. Pública, que promueva la modernización y el cambio en las empresas en la línea del Modelo propuesto. 


\section{Grupo II. Posibles cambios normativos y legales}

1. Aprovechar las ayudas a las empresas para impulsar un Modelo inclusivo participativo más moderno en las mismas.

2. Desarrollar el Reglamento de Empresas Participadas, recogiendo en el mismo las características del Modelo inclusivo participativo propuesto y creando el Registro de Empresas Participadas que pueda ayudar a dar seguridad jurídica a las medidas que se arbitren en su promoción.

3. Impulsar un nuevo Estatuto de la Empresa Pública que aúne eficiencia y participación.

4. Estudiar una fórmula jurídica adecuada y, en su caso, la elaboración de una ley que facilite a los trabajadores el acceso a la propiedad de la empresa en aquellos casos en que éstas lo consideren adecuado para la sostenibilidad y/o el desarrollo de su proyecto empresarial (empresas familiares con problemas de sucesión, start ups, empresas en crisis con viabilidad, empresas intensivas en conocimiento...), priorizando a los mismos frente a otros posibles inversores a fin de reforzar, a la vez, el arraigo territorial de las empresas.

5. Modificar la ley concursal para facilitar la continuidad de las empresas, preferentemente mediante fórmulas de participación de los trabajadores.

6. Estudiar una ley u otra fórmula alternativa para el desarrollo de la fórmula de las ESOP de USA.

7. Estudiar que las aportaciones de los trabajadores al capital de la empresa en que trabajan tengan el mismo tratamiento fiscal que las aportaciones que realizan a los Planes de Pensiones.

8. Dar un salto cualitativo en la dinámica empresarial mediante la capitalización de ajustes salariales.

\section{Conclusiones}

La Economía Social vasca goza de un merecido prestigio en Europa $y$, nos atreveríamos a decir, que también en el mundo. El Grupo Mondragón es un referente del cooperativismo mundial y paradigma de éxito empresarial.

Las Sociedades Laborales han supuesto una innovación societaria, de las que no se registran muchas en Europa. En ambos casos se comprueba que han sido capaces de expandirse aprovechando las coyunturas económicas favorables y de mostrar una especial resiliencia cuando son adversas. 
Pero casi dos siglos de cooperativismo en el mundo y medio siglo de Sociedades laborales también permiten vislumbrar los límites de las fórmulas jurídicas de lo que podríamos denominar «emprendimiento colectivo». (Paradójicamente, son las mismas cooperativas las que utilizan fórmulas societarias convencionales cuando salen fuera de su comunidad natural).

Desde otro punto de vista, numerosos estudios (recogidos algunos de ellos en estas mismas páginas) muestran que los valores de la Economía Social llevan a prácticas de gestión que consiguen una mayor identificación de las personas con el proyecto empresarial y un mejor aprovechamiento de sus capacidades y potencialidades humanas al servicio del proyecto colectivo, que tienen como consecuencia una mayor competitividad y sostenibilidad a largo plazo de las empresas.

Colaborar en «inocular» esos valores y prácticas de gestión en empresas convencionales (siquiera con una menor intensidad) puede ser una aportación adicional importante para la Economía Social, aplicando así la máxima de Arizmendiarrieta de Transformar la empresa para transformar la sociedad. El Modelo inclusivo participativo propuesto puede ser una fórmula adecuada para ello. 